\title{
Approach to Malignant Bowel Obstruction in Palliative Care
}

\author{
Sue Wern Neoh ${ }^{*}$ \\ Department of Palliative Medicine, Ng Teng Fong General Hospital, Singapore
}

*Corresponding author: Sue Wern Neoh, Department of Palliative Medicine, Ng Teng Fong General Hospital, Singapore, Tel: +65-67162000; E-mail: sue_wern_neoh@juronghealth.com.sg

Received date: September 26, 2016, Accepted date: October 25, 2016, Published date: November 01, 2016

Copyright: (c) 2016 Neoh SW. This is an open-access article distributed under the terms of the Creative Commons Attribution License, which permits unrestricted use, distribution, and reproduction in any medium, provided the original author and source are credited.

\begin{abstract}
Malignant bowel obstruction is a frequent complication in patients with advanced cancer. Based on a case presentation this article will highlight a palliative medicine approach to addressing this distressing complaint. Focusing primarily on the prevailing treatment options of malignant bowel obstruction at the end of life, this article also evaluates decision making process behind the care of malignant bowel obstruction and highlights a patientcentered care.
\end{abstract}

Keywords: Malignant bowel obstruction; Palliative medicine; End of life

\section{Introduction}

Treatment of malignant bowel obstruction (MBO) is complex and it requires understanding of the pathophysiology of bowel obstruction, careful assessment and decision for the best treatment option. Importantly treatment of MBO requires a holistic and patient specific approach. This case discussion will set out to highlight the complex nature of holistic care of malignant bowel obstruction in a palliative care setting.

\section{Case Report}

\section{Background}

Patient is a 62-year-old woman who was diagnosed with stage IV gastric adenocarcinoma with metastasis to peritoneum and anterior abdominal wall. She had a palliative distal gastrectomy, followed by chemotherapy. Unfortunately, a repeat computed tomography (CT) scan after 2 cycles of chemotherapy showed progression of disease in peritoneum and abdominal wall.

\section{Current admission}

She presented with vomiting and abdominal colic for 2 days, but was still able to pass small amount of stool daily. She had vomiting 3 to 4 episodes per day which was unrelated to meals. The vomitus was bilious with partially digested food. On examination, she was not dehydrated; her abdomen was distended but soft. Bowel sound was sluggish. There were also palpable nodules on the abdominal wall which were mildly tender.

An urgent CT scan was arranged. It showed a small bowel obstruction with a transition point at proximal jejunum. In view of persistent vomiting, a nasogastric tube was inserted. She was told that the nasogastric tube insertion would be a temporary measure for decompression.
As patient was still opening her bowels the impression was a partial rather than complete bowel obstruction. The intention of management was to reverse the obstruction. She was given a suppository bisacodyl, regular intravenous metoclopramide $10 \mathrm{mg}$ every 8 hour and intravenous dexamethasone $8 \mathrm{mg} /$ day. She was also started on fentanyl infusion $10 \mathrm{mcg} / \mathrm{hr}$ for worsening of nociceptive lower abdominal pain from the peritoneal metastasis that had been present for two months.

With no ill effects but no bowel action, the intravenous metoclopramide was increased to $60 \mathrm{mg} /$ day on the following day.

There was marked clinical improvement 2 days later. The NG aspirate has reduced significantly and she had no more vomiting. The team doctor was able to remove her nasogastric tube and started her on oral feed gradually. Intravenous metoclopramide and dexamethasone were continued for two days and stopped subsequently.

Three days later, she started to vomit again. She had 3 episodes of vomiting in a day which contains moderate to large amount of bilious vomitus. She was recommenced on intravenous metoclopramide 60 $\mathrm{mg}$ per day and dexamethasone $8 \mathrm{mg}$ per day. In addition intravenous ranitidine $150 \mathrm{mg}$ bd was added to reduce gut secretions.

Despite the above treatment, her symptoms persisted. She declined having a nasogastric tube re-inserted due to the previous unpleasant experience. After discussing with patient and her family members, her team doctor decided to start her on intravenous octreotide $100 \mathrm{mcg}$ tds, which was then increased to $200 \mathrm{mcg}$ tds the following day. At the same time, she was arranged for a gastrografin study which showed dilated small bowel predominantly jejunum loops with increased transit time from duodenum to colon. There was no transition point seen and a surgical intervention was not deemed viable. Her symptoms resolved 2 days after the combination of the above measures.

As she continued to improve, preparations were made to fulfill her wish of going home as soon as possible. This includes switching the essential parenteral medications to oral route, tailing down dexamethasone dose, and plans to start on long-acting monthly injectable octreotide.

Unfortunately, she developed similar episode of bowel obstruction again one week later. Her team doctor managed to convince her for 
nasogastric tube insertion. Dexamethasone and metoclopramide dose were increased back to $8 \mathrm{mg}$ and $60 \mathrm{mg}$ per day respectively, delivered parenterally, and octreotide was continued. However, treatment was not successful this time, the nasogastric aspirate remained high. She was given options of having a venting gastrotomy or keeping the nasogastric tube as removal of the tube would likely lead to intractable vomiting again.

Goal of care was established after discussing with patient and her family. She preferred to keep the nasogastric tube for decompression while she enjoyed taking fluid orally. Decision was also made to stop further chemotherapy and for comfort care. She returned home soon after that with home hospice support.

\section{Discussion and Focused Literature Review}

To facilitate a meaningful discussion on the clinical considerations facing, her family and the team caring for her, a better understanding of the pathophysiology of $\mathrm{MBO}$ is required.

\section{Pathophysiology of malignant bowel obstruction}

Malignant bowel obstructions can be categorized based on:

- Extent: partial or complete; single level or multilevel

- Aetiology: mechanical or functional

- Site: proximal or distal bowel

Malignant bowel obstruction ( $\mathrm{MBO}$ ) may occur any time along the disease trajectory though most frequently in advanced cancer. The causes of $\mathrm{MBO}$ are largely divided into a) factors directly related to intra-abdominal tumor growth such as extrinsic intestinal compression, endoluminal intestinal obstruction, intraluminal intestinal infiltration and infiltration of mesentery or plexus, b) factors not directly related to intra-abdominal tumor growth such as paraneoplastic neuropathy, adynamic ileum, postsurgical adhesion, opioid-induced intestinal dysfunction or inflammatory intestinal disease [1].

In intestinal obstruction, fluid retention and intestinal gases proximal to the occlusion causes increase in the intestinal pressure which leads to activation of the enteric interneuronal system and stimulation of secretomotor neurons which are especially mediated by vasoactive intestinal peptide (VIP). This will then lead to splanchnic vasodilatation and hypersecretion which leads to further increases in the endoluminal pressure $[1,2]$.

In case of this patient, she had recurrent partial small bowel obstructions at multiple sites along the bowel as a result of either carcinomatosis peritonei or adhesions as a result of her previous partial gastrectomy.

\section{Decision making in the management of malignant bowel obstruction}

The decision making process for the management of $\mathrm{MBO}$ requires careful consideration of multiple factors inclusive of the patient's wishes, goals of care, practical considerations surrounding overall care as well as due consideration of the level and aetiology of obstruction, clinical stage of cancer and prognosis, future anti-cancer treatment, patient's health and performance status and feasibility of surgical intervention [2]. These are summarized into patient factors, disease factor and treatment/technical factors (Figure 1).
Patients with good performance status, early stages of disease and single level bowel obstruction favour surgical intervention. The presence of significant ascites, multilevel obstruction and carcinomatosis peritonei often negates the possibility of surgery.

In carcinomatosis peritonei, the obstruction is usually partial, intermittent and occurs at multiple levels of small and large bowel, possibly due to motility disorders secondary to bowel wall infiltration by tumor and/or involvement of parasympathetic nerve responsible for peristalsis. Surgery for patients with carcinomatosis peritonei therefore is associated with poor outcome and high recurrence rate. Similarly it was not indicated in this case.

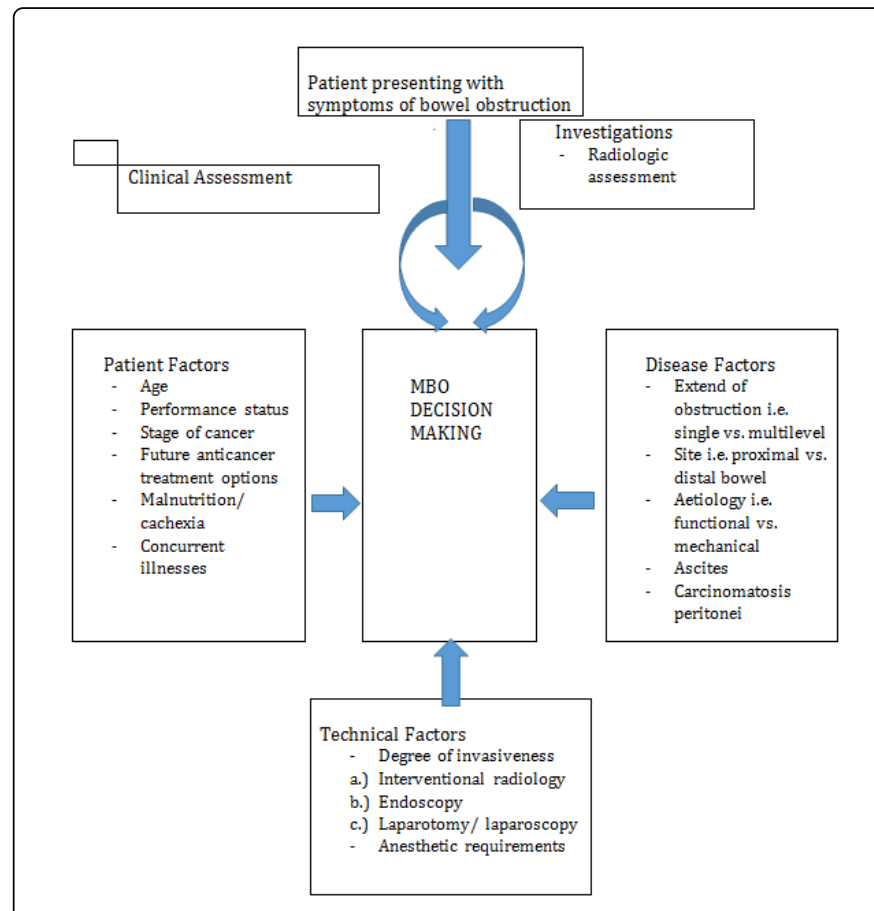

Figure 1: Algorithm for assessing and managing a patient with malignant bowel obstruction [2].

\section{Pharmacological treatment of malignant bowel obstruction}

The mainstay of non-surgical palliation of malignant bowel obstruction includes analgesia, anti-emetic and measures aimed at reducing intestinal secretions.

Analgesia: In patients with bowel obstruction, there are usually 2 types of pain: a) abdominal colic as a result of giant peristaltic waves and bowel spasms with associated raised luminal pressure and poor transit of intestinal content. b) persistent nociceptive pain from tumour infiltration of the abdominal structures and visceral distension with gas and fluids. According to the European Society of Palliative Care and the World Health Organisation (WHO), morphine is the opioid of choice for moderate to severe cancer pain $[3,4]$. There have been no controlled clinical trials comparing different opioids in bowel obstruction so far. Studies have showed that fentanyl produces less constipation than morphine [5]. This property, in addition to its availability as a transdermal formulation, makes it useful in management of MBO. 
In this case, fentanyl was chosen by her physician simply due to convenience as she was already on fentanyl patch for her nociceptive abdominal pain from the peritoneal disease. She had abdominal colic initially which then resolved spontaneously. Instead, there was worsening of constant nociceptive pain requiring fentanyl infusion for faster titration for pain control. In patients with intestinal obstruction or those at high risk of obstruction, oral route of analgesia is to be avoided to ensure effective absorption of the medications. This has impact upon care determinations given that care at home may be difficult in the face of recurrent episodes of pain and the need for injections.

Corticosteroids: Steroids have been successfully used for their antiinflammatory effects in many conditions such as intracranial hypertension, spinal cord compression, as well as inoperable bowel obstruction. In intestinal obstruction, corticosteroids work centrally as potent analgesia and anti-emetic as well as peripherally in reducing peritumoural inflammatory oedema and increasing water and salt absorption in the gut [6]. Laval et al. [7] performed a randomised controlled trial and demonstrated that significantly higher number of patients without nasogastric tube and treated with steroid had resolution of bowel obstruction compared to those receiving placebo. There are also other studies that show effectiveness of intravenous dexamethasone of dose range $6 \mathrm{mg}$ to $16 \mathrm{mg}$ in resolving bowel obstruction [6]. The use of steroids is also further supported by their tolerability, low incidence of side effects and low cost.

Anti-emetic: Nausea and vomiting in bowel obstruction can be managed pharmacologically by reducing the gastrointestinal secretion and working on the emetogenic pathway. Anti-emetics largely targets central and/or peripheral dopaminergic, serotonergic and acetylcholine receptors. Metoclopramide is primarily a dopamine antagonist and also serotonin type 4 receptor agonist. Its additional prokinetic property makes it an effective drug in functional and partial bowel obstruction. It was aimed to help with motility of intestine and to reverse the obstruction with the combination of other drugs mentioned below.

Her symptom improved markedly with metoclopramide and dexamethasone at the first episode of partial bowel obstruction. The patient did not develop abdominal colic along the course of her treatment.

In complete bowel obstruction or presence of abdominal colic, metoclopramide should be avoided, and haloperidol is considered. Haloperidol is a butyrophenone selective dopamine D2-receptor antagonist, with negligible anticholinergic activity, which has profound inhibitory effect on the chemoreceptor trigger zone. Doses range from $5 \mathrm{mg} /$ day to $15 \mathrm{mg} /$ day, given in divided doses or continuous intravenous infusions [8].

The serotonin (5-HT3) receptor antagonists used in MBO include ondansetron or granisetron. Anti-cholinergic such as hyoscine butylbromide, hyoscine hydrobromide and glycopyrrolate is another group of anti-emetic which has also anti-secretory properties.

Anti-secretive drugs: Successful use of octreotide, a somatostatin analogue, in inoperable malignant bowel obstruction has been reported since early 1990's [9]. Octreotide reduces splanchnic blood flow and inhibits release of gastrin, vasoactive intestinal peptide and pancreatic polypeptide in addition to its endocrine action of reducing growth hormone and insulin release. It reduces intestinal fluid secretion and improves water and sodium absorption from the gut, leading to break of vicious cycle of distension and secretion in bowel obstruction.

Octreotide has a longer duration of action of up to 12 hours and is more potent than the natural compound somatostatin. The peak plasma concentration of octreotide occurs 30 minutes after administration and its half-life is around 1.5 hours. It can be administered as intravenous or subcutaneous injection. This drug is well tolerated without significant side effects. Reports of pain at the injection site with minor skin reaction are rare.

In case of this patient, her symptom was controlled with anti-emetic and corticosteroid in the first episode of bowel obstruction. However, this conventional treatment failed to control her symptoms when she had recurrent obstruction. Thus decision was made to add octreotide. It was started at $0.3 \mathrm{mg} /$ day and titrated up to $0.6 \mathrm{mg} /$ day subsequently. The frequency of vomiting improved significantly and she was able to take orally again.

Another commonly used anti-secretive drug is hyoscine butylbromide. Octreotide was shown to be more effective than hyoscine butylbromide in controlling vomiting and nausea [10-12]. Ripamonti et al. [13] did a prospective, randomised clinical trial on 17 patients and found that octreotide was superior to scopolamine in reducing GI secretions and intensity of nausea in the home care patients. This can be explained by the fact that octreotide has a potent anti-VIP effect that results in inhibition of intestinal secretion, which is the principal mechanism in bowel obstruction. However, as octreotide is expensive, physicians need to weigh its cost-benefit ratio carefully, especially for prolonged treatment.

For patients who respond to short-acting octreotide, intramuscular long-acting octreotide can be considered for subsequent treatment, which is administered monthly. Besides the convenience of monthly injection, long-acting octreotide also has attractive advantage when there is concern about limited absorption of subcutaneous short-acting octreotide in patients with oedema and poor peripheral blood flow. Patients usually require short-acting octreotide for the first 2 weeks because long-acting octreotide doesn't reach therapeutic levels until at least 14 days after injection [14].

Histamine 2 Antagonist: Besides hyoscine butylbromide and octreotide, histamine 2 antagonists and proton pump inhibitor that work on gastric parietal cells also reduce gastric secretions and may be considered in management of bowel obstruction. Meta-analysis reported that histamine 2 antagonists reduce volume of gastric secretion to a significantly greater degree than proton pump inhibitor $[15,16]$. The studies were carried out in patients who were fasting and undergoing low risk anaesthesia. The evidence of histamine 2 antagonist in malignant bowel obstruction is still lacking and further clinical studies are required.

Contrast Medium: According to Mercadante et al. [17], amidotrizoato or gastrografin, a hyperosmolar water-soluble contrast medium, has a therapeutic role in adhesive, partial bowel obstruction. It has osmolarity of $1900 \mathrm{mOsm} / \mathrm{L}$, approximately six times that of extracellular fluid. It promotes shifting of fluid into the bowel lumen thus diluting and facilitating the passage through a narrower lumen. This substance also decreases oedema of the bowel wall and enhances the intestinal motility. Study has shown that gastrografin is safe to use and reduces the need for surgery when conservative treatment fails [18]. 
She had a gastrografin meal and follow through for diagnostic and therapeutic purposes. The test findings helped provide an idea of the anatomy and the likelihood of surgery. In this case, it was carried out when the initial analgesia, anti-emetic and corticosteroids failed to relieve her symptoms. Together with the combination of the above medications and octreotide, resolution of the partial bowel obstruction was achieved.

Another commonly used contrast, barium, is not as easily diluted by enteric fluid as gastrografin and provides a better mucosal image on radiography. However, gastrografin is thought to be a safer choice as barium may become inspissated and completely obstruct the bowel. Moreover, barium may spread into the peritoneal cavity if perforation occurs, which can be life-threatening. Gastrografin, on the other hand, is water-soluble and relatively safer if the bowel obstruction is complicated by perforation [18].

\section{Conclusion}

Malignant bowel obstruction is a distressing condition which is common among patient with advanced cancer and the underlying aetiology is often multifactorial. Although surgery or endoscopic stenting are effective in reversing bowel obstruction, they are often not suitable options in our group of patients in palliative medicine. This case report presents the evidence based approach of pharmacological treatment in malignant bowel obstruction. It also highlights that $\mathrm{MBO}$ may not resolve despite aggressive medical treatment when there is complete or recurrent bowel obstruction. Constant discussion with patients is essential to meet their expectations and to establish goal of care. In this case presentation, when the bowel obstruction was recurrent and unresolved, patient's goal was switched to comfort care and arrangement was made to fulfill her wish of spending time with family at home.

\section{References}

1. Tuca A, Guell E, Martinez-Losada E, Codorniu N (2012) Malignant bowel obstruction in advanced cancer patients: epidemiology, management, and factors influencing spontaneous resolution. Cancer Manag Res 4: 159-169.

2. Ripamonti CI, Easson AM, Gerdes H (2008) Management of malignant bowel obstruction. Eur J Cancer 44: 1105-1115.

3. Hanks GW, Conno F, Cherny N, Hanna M, Kalso E, et al. (2001) Morphine and alternative opioids in cancer pain: the EAPC recommendations. Br J Cancer 84: 587-593.

4. WHO (1996) Cancer Pain Relief - with a guide to opioid availability. 2nd edn, World Health Oranization, Geneva, Switzerland.
5. Tassinari D, Sartori S, Tamburini E, Scarpi E, Tombesi P, et al. (2009) Transdermal fentanyl as a front-line approach to moderate-severe pain: a meta-analysis of randomized clinical trials. J Palliat Care 25: 172-180.

6. Feuer DJ, Broadley KE (2000) Corticosteroids for the resolution of malignant bowel obstruction in advanced gynaecological and gastrointestinal cancer. Cochrane Database Syst Rev 2: CD001219.

7. Laval G, Girardier J, Lassauumiere JM, Leduc B, Haond C, et al. (2000) The use of steroids in the management of inoperable intestinal obstruction in terminal cancer patients: do they remove the obstruction? Palliat Med 14: 3-10.

8. Soriano A, Davis MP (2011) Malignant Bowel Obstruction: Individualised treatment near the end of life. Cleve Clin J Med 78: 197-206.

9. Mercadante S, Spoldi E, Caraceni A, Maddaloni S, Simonetti MT (1993) Octreotide in relieving gastrointestinal symptoms due to bowel obstruction. Palliat Med 7: 295-299.

10. Mystakidou K, Tsilika E, Kalaidopoulou O, Chondros K, Georgaki S, et al. (2002) Comparison of octreotide administration vs conservative treatment in the management of inoperable bowel obstruction in patients with far advanced cancer: a randomised, double-blind, controlled clinical trial. Anticancer Res 22: 1187-1192.

11. Mercadante S, Ripamonti C, Casuccio A, Zecca E, Groff L (2000) Comparison of octreotide and hyoscine butylbromide in controlling gastrointestinal symptoms due to malignant inoperable bowel obstruction. Support Care Cancer 8: 188-191.

12. Mercadante S, Casuccio A, Mangione S (2007) Medical Treatment for inoperable malignant bowel obstruction: A qualitative systematic review. J Pain Symptom Manage 33: 217-223.

13. Ripamonti C, Mercadante S, Groff L, Zecca E, De Conno F, et al. (2000) Role of octreotide, scopolamine butylbromide and hydration in symptom control of patients with inoperable bowel obstruction having a nasogastric tube - a prospective, randomized clinical trial. J Pain Symptom Manage 19: 23-34.

14. Matulonis UA, Seiden MV, Roche M, Krasner C, Fuller AF, et al. (2005) Long-Acting Octreotide for the Treatment and Symptomatic Relief of Bowel Obstruction in Advanced Ovarian Cancer. J Pain Symptom Manage 30: 563-569.

15. Clark K, Lam L, Currow D (2009) Reducing gastric secretions-a role for histamine 2 antagonists or proton pump inhibitors in malignant bowel obstruction? Support Care Cancer 17: 1463-1468.

16. Clark K, Lam LT, Gibson S, Currow D (2009) The effect of ranitidine vs proton pump inhibitors on gastric secretions: a meta-analysis of randomised control trials. Anaesthesia 64: 652-657.

17. Mercadante S, Ferrera P, Villari P, Marrazzo A (2004) Aggressive pharmacological treatment for reversing malignant bowel obstruction. J Pain Symptom Manage 28: 412-416.

18. Choi HK, Chu KN, Law WL (2002) Therapeutic Value of Gastrografin in Adhesive Small Bowel Obstruction After Unsuccessful Conservative Treatment. A Prospective Randomised Trial. Ann Surg 6: 1-6. 